\title{
CSP01, a Novel Superabsorbent Hydrogel, Reduces Colonic Transit Time in Patients With Chronic Idiopathic Constipation in a Randomized, Double-blind, Controlled Pilot Clinical Trial
}

\author{
Kyle Staller, ${ }^{1 *}$ Kenneth Barshop, ${ }^{2}$ Christopher Vélez, ${ }^{1}$ Abbey Bailey, ${ }^{1}$ Joseph J Locascio, ${ }^{3}$ Elaine Chiquette, ${ }^{4}$ and Braden Kuo ${ }^{1}$ \\ ${ }^{I}$ Division of Gastroenterology, Massachusetts General Hospital and Harvard Medical School, Boston, MA, USA; ${ }^{2}$ Division of Gastroenterology, \\ Beth Israel Deaconess Medical Center, Boston, MA, USA; ${ }^{3}$ Department of Neurology, Massachusetts General Hospital, Boston, MA, USA; and \\ ${ }^{4}$ Gelesis Inc, Boston, MA, USA
}

\section{Background/Aims}

CSP01 is a novel superabsorbent hydrogel that absorbs gastrointestinal fluids and maintains high viscoelastic properties into the colon, where these fluids are released.

\section{Methods}

We conducted a single-center, randomized, double-blind, parallel-group, placebo-controlled pilot study comparing change in colonic transit time (CTT) among patients with chronic idiopathic constipation (CIC) and irritable bowel syndrome with constipation (IBS-C) treated for 21 days with either CSP01 hydrogel, active control (carboxymethylcellulose [CMC]) or placebo. CTT was measured using wireless motility capsule transit testing at pre-treatment and end-of-treatment. The primary endpoint was change in CTT.

\section{Results}

Forty subjects (20 CSP01, 11 CMC, 9 placebo) were enrolled and 38 completed the study. There was no significant change in mean CTT by treatment group $(P=0.297)$. In the placebo group, CTT increased by 15.3 minutes between baseline and end of treatment, increased by 366.4 minutes for CMC, and decreased by 727.4 minutes for CSP01. In post hoc analyses among those with $\mathrm{CIC}$, mean CTT decreased by 1079 minutes for CSP01 ( $P=0.025$ compared to placebo), 919 minutes for CMC ( $P=0.117$ compared to placebo) and increased by 1113 minutes for placebo. Among patients with IBS-C, there was no significant difference in change in CTT for any treatment group. One subject in the CSP01 arm developed back pain attributed to constipation and withdrew without a second CTT measurement; there were no other adverse events.

\section{Conclusion}

CSP01 significantly decreased CTT compared to placebo among patients with CIC, but not in patients with IBS-C.

(J Neurogastroenterol Motil 2020;26:496-504)

Key Words

Capsules; Constipation; Gastrointestinal motility; Hydrogels; Irritable bowel syndrome

Received: January 3, 2020 Revised: June 5, 2020 Accepted: June 15, 2020

(.) This is an Open Access article distributed under the terms of the Creative Commons Attribution Non-Commercial License (http://creativecommons. org/licenses/by-nc/4.0) which permits unrestricted non-commercial use, distribution, and reproduction in any medium, provided the original work is properly cited.

*Correspondence: Kyle Staller, MD, MPH

Division of Gastroenterology, Massachusetts General Hospital and Harvard Medical School, 55 Fruit Street, Boston, MA 02114, USA Tel: +1-617-724-6038, Fax: +1-617-724-5996, E-mail: kstaller@mgh.harvard.edu 


\section{Introduction}

Chronic constipation is a highly-prevalent condition affecting approximately $14 \%$ of the population globally, with substantial impact on quality of life. ${ }^{2}$ Unlike other chronic diseases, treatment options for chronic constipation are frequently available without a prescription in the form of fiber supplements, laxatives, and herbal preparations.

Increased intake of dietary fiber or fiber supplements is often the initial recommendation for treatment of constipation, though high-quality evidence supporting its use is minimal. Previous trials using fiber supplementation in constipation have focused on a variety of subjective, symptom-based outcomes without evaluation of physiologic effects. ${ }^{3}$ Some fibers have demonstrated specific effect in irritable bowel syndrome (IBS) ${ }^{4}$ with others showing benefit in constipation. ${ }^{5}$ A previous, short-term trial employing wheat dextrin demonstrated a reduction in colonic transit time (CTT) among healthy controls after 3 days of treatment. ${ }^{6}$ Despite multiple overthe-counter options, there remains a need for more effective and well-tolerated therapeutic options.

CSP01 (Gelesis Inc, Boston, MA, USA) is a novel superabsorbent hydrogel synthesized from a commonly used soluble fiber, carboxymethylcellulose (CMC), cross-linked with citric acid. The resultant 3-dimensional (3D) structure swells and absorbs 85 times its weight in water. As a result, CSP01 no longer has the properties of CMC: (1) its water-holding capacity is significantly higher, (2) its "solid-like gel" shape travels intact through the gut, and (3) it exerts more elastic response (firmness) than conventional fibers. ${ }^{8} \mathrm{CSP} 01$ capsules contain superabsorbent hydrogel particles that swell to form individual gel pieces that mix homogeneously with food in the stomach, and retain their 3D structure throughout the small intestine before degradation in the colon with release of absorbed fluids. We hypothesized that CSP01 may accelerate gastrointestinal (GI) transit because of its unique mechanical properties and thus aimed to determine the effect of CSP01 on CTT compared to an active control CMC (backbone of CSP01) and placebo in a double-blind, placebo-controlled randomized pilot study.

\section{Materials and Methods}

\section{Study Design, Participants, and Medications}

This study was a single-site, double-blind, placebo-controlled, parallel-group randomized study evaluating the pharmacodynamic effects of CSP01 (2.37 g), CMC (active control, $2.10 \mathrm{~g}$ ), and placebo administered orally twice daily for 21 days among patients with chronic idiopathic constipation (CIC) and IBS with constipation (IBS-C) according to the Rome IV criteria. ${ }^{9}$ Subjects were recruited from the gastroenterology clinic at Massachusetts General Hospital as well as from targeted, online advertisements in the local community. The study was approved by the institutional review board of Massachusetts General Hospital (IRB No. 2016P001751) and registered with ClinicalTrials.gov (NCT03054506). All patients signed informed consent. Patients were allowed to continue all medications except for laxatives or intestinal secretagogues. Exclusion criteria included colonic inertia ( $<1$ bowel movement/14 days), any structural or metabolic diseases/conditions that affect the GI system, or medication-induced constipation.

The study consisted of 3 periods: run-in, treatment, and post treatment follow-up (Fig. 1). After a screening visit in which patient eligibility was determined through use of Rome IV constipation

\begin{tabular}{|c|c|c|c|c|c|c|c|c|}
\hline \multirow[b]{2}{*}{ Time point (day) } & \multirow{2}{*}{$\begin{array}{l}\text { Screening } \\
-20 \text { to }-15\end{array}$} & \multicolumn{3}{|c|}{ Run-in period } & \multicolumn{3}{|c|}{$\begin{array}{l}\text { Treatment period } \\
\text { CSP01 or } \\
\text { CMC or } \\
\text { Placebo }\end{array}$} & \multirow[b]{2}{*}{31} \\
\hline & & -14 & -7 & 0 & 1 & 15 & 22 & \\
\hline \multicolumn{9}{|l|}{ Efficacy } \\
\hline Rome IV screen & $\bullet$ & & & $\bullet$ & & & & \\
\hline PAC-SYM & $\bullet$ & $\bullet$ & & $\bullet$ & $\bullet$ & $\bullet$ & $\bullet$ & \\
\hline PAC-QOL & & $\bullet$ & & $\bullet$ & $\bullet$ & $\bullet$ & $\bullet$ & \\
\hline Daily diary & & & & & & & & $\rightarrow$ \\
\hline Safety & & & & & & & & $\rightarrow$ \\
\hline WMC testing & & & $\bullet$ & & & $\bullet$ & & \\
\hline Adverse events & & & & & $\bullet$ & $\bullet$ & $\bullet$ & $\bullet$ \\
\hline
\end{tabular}

Figure 1. Study design. CMC, carboxymethylcellulose; WMC, wireless motility capsule; PAC-SYM, Patient Assessment of Constipation-Symptoms; PAC-QOL, Patient Assessment of Constipation-Quality of Life. 
module along with other inclusion/exclusion criteria, eligible patients enrolled in a 14-day run-in period. Patients completed a daily stool diary (Day -14 to Day 0) to ensure they continued to meet criteria for CIC or IBS-C and underwent wireless motility capsule (WMC) testing (Day -7 to Day 0) to assess baseline CTT. If still meeting inclusion criteria, patients were randomized (simple randomization) to 1 of the 3 groups: CSP01 $2.10 \mathrm{~g}$, matching CMC $2.37 \mathrm{~g}$ (corresponding to the amount of $\mathrm{CMC}$ hydrogel precursor contained in the CSP01 dose), or matching placebo. Thus, the agents differed primarily on their rheological properties (inherent material properties that govern elastic response to an applied force) with a similar CMC backbone. The randomization code was generated by the Massachusetts General Hospital research pharmacy, with study medication distributed to study staff in a blinded fashion. All study personnel and patients were blinded throughout the study until database lock and data analyses. For the following 21 days of treatment, patients took 3 capsules of either placebo, CMC or CSP01 twice a day (before breakfast and dinner) with 2 glasses of water (16 oz) and completed a daily stool diary.

A second WMC test was done on Day 15 to Day 21 of the treatment period. The patient completed daily stool diaries and adverse event (AE) monitoring for an additional $7 \pm 3$ days (Day 22 to Day 31) after the last dose of treatment. Safety monitoring was conducted throughout the study.

\section{Colonic Transit Testing and Analysis}

The primary endpoint was the change in CTT from pretreatment (Day -7 to Day 0) to last week of treatment (Day 15 to Day 21). We used the established WMC technique (SmartPill; Medtronic plc, Fridley, MN, USA), a reliable means of measuring $\mathrm{CTT}^{10,11}$ previously used in a clinical trial to assess treatment with fiber. ${ }^{6}$ CTT was defined as the time interval between the points of entry into the cecum and capsule exit from the body. CTT $\geq 59$ hours is classified as slow-transit constipation.

\section{Daily Stool Diaries}

Each patient noted the exact time of each bowel movement during the run-in and treatment periods along with the following descriptions: stool consistency according to the Bristol stool form scale (ranging from 1, "hard lumps" to 7, "watery"), ${ }^{12,13}$ ease of passage (ranging from 1, "manual disimpaction” to 7, "incontinence"), and answered whether or not they felt completely evacuated (yes/ no). Additionally, patients completed daily ratings of global constipation severity, bloating severity, and abdominal discomfort severity, all assessed on a 0-10 scale (higher values indicate more severe symptoms). Finally, patients completed the Patient Assessment of Constipation-Symptoms (PAC-SYM) ${ }^{14}$ and the Patient Assessment of Constipation-Quality of Life (PAC-QOL) ${ }^{15}$ at the beginning of the run-in period (Day -14) and on Days 0, 15, and 22 of the treatment period.

\section{Statistical Methods}

For the primary outcome, change in CTT from baseline to Day 21 of treatment, we employed a modified intention-to-treat analysis where all patients with pre- and post-treatment CTT data were included in the analysis. In cases where the primary outcome was unavailable but secondary outcome information was available, we performed an intention-to-treat analysis as planned prior to study initiation. In order to assess the effects of treatment (CSP01 vs CMC vs Placebo) on CTT, we employed a mixed between subject and within subject repeated measures analysis of covariance (ANCOVA). Between subject factors selected based on clinical relevance were treatment group, diagnosis (CIC or IBS-C), gender, and age, whereas the within subject factor crossed with each of the above was a 2-level time factor (7 days pre-treatment or Day 15 of treatment). In addition, the interactions of treatment with gender and with IBS were also tested (and each further crossed with time). A backward elimination algorithm was applied to the full saturated model. Post hoc analyses specifically examined CTTs among patients with CIC vs IBS. For the secondary outcomes, the analysis of the daily diary data, we first computed the mean scores per 2-week period (baseline and last 2 weeks of active treatment) in each subject. Between subject factors selected based on clinical relevance were treatment group, Rome IV diagnosis (CIC or IBSC), gender, and age, whereas the within subject factor crossed with each of the above was a 2-level time factor (average scores over 2 weeks of pre-treatment or over the last 2 weeks of active treatment). Similar analyses were performed with the addition of baseline slowtransit constipation (baseline CTT $\geq 59$ hours) to the betweensubject factors in the group as a whole and with IBS status factored in. Statistical analyses were performed using SAS version 9.4 (SAS Institute, Cary, NC, USA).

\section{Sample Size}

Sample sizes planned for the study were based on mean and coefficient of variation data from a prior study evaluating the effects of fiber on CTT using the WMC. ${ }^{6}$ From this previous data, there was a 10-hour difference in CTT with a standard deviation of 12 hours, which we assumed would be similar to the effect of our active control CMC group. Assuming that CSP01 would give an additional 
3-hour decrease in CTT over conventional fiber, we anticipated enrolling 15 subjects per arm where the calculated sample size would have $94 \%$ power to detect a significant difference at an alpha level of
0.05. More conservatively, with 10 subjects per arm, we would have $80 \%$ power to detect a significant difference between groups.

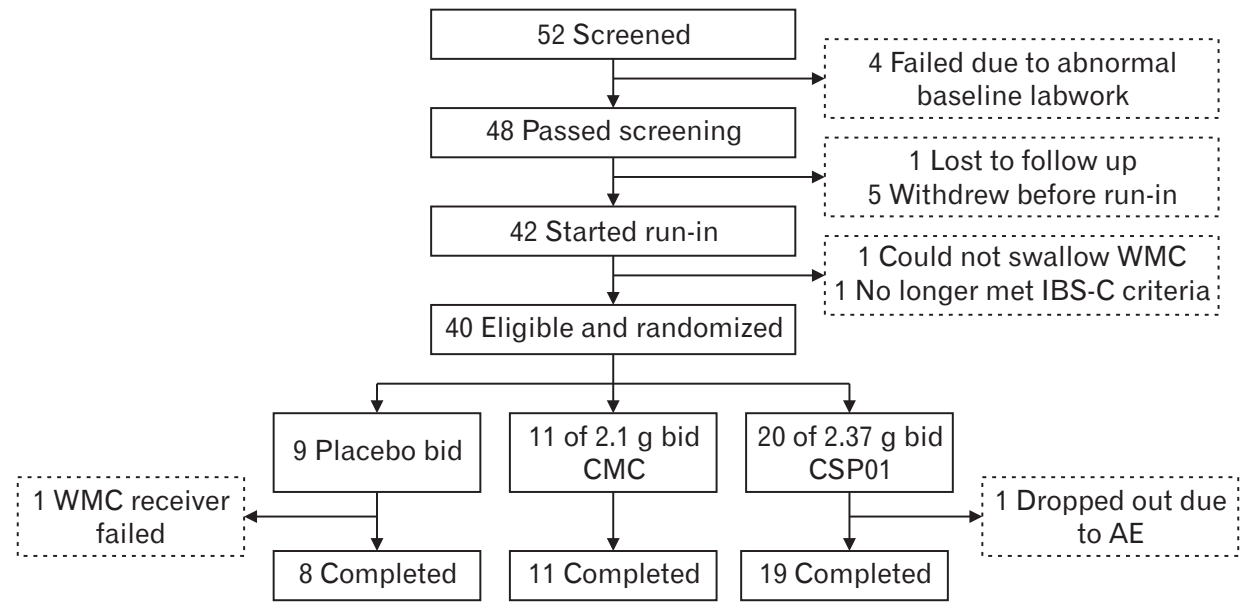

Figure 2. Study flow chart. WMC, wireless motility capsule; IBS-C, irritable bowel syndrome with constipation; bid, twice a day; CMC, carboxymethylcellulos; AE, adverse event.

Table 1. Demographic and Baseline Characteristics, Intention to Treat Population

\begin{tabular}{|c|c|c|c|}
\hline \multirow[b]{2}{*}{ Patient characteristics } & \multicolumn{3}{|c|}{ Treatment assignment } \\
\hline & $\begin{array}{l}\text { Placebo } \\
(\mathrm{n}=9)\end{array}$ & $\begin{array}{l}\text { Carboxymethylcellulose } \\
\qquad(\mathrm{n}=11)\end{array}$ & $\begin{array}{l}\text { CSP01 } \\
(n=20)\end{array}$ \\
\hline Age (yr) & $51.9 \pm 12.7$ & $41.5 \pm 17.7$ & $44.9 \pm 18.1$ \\
\hline \multicolumn{4}{|l|}{ Sex } \\
\hline Female & $7(77.8)$ & $9(81.8)$ & $19(95.0)$ \\
\hline Male & $2(22.2)$ & $2(18.2)$ & $1(5.0)$ \\
\hline \multicolumn{4}{|l|}{ Race } \\
\hline White & $8(88.9)$ & $8(72.7)$ & $16(80.0)$ \\
\hline Non-white & $1(11.1)$ & $3(27.3)$ & $4(20.0)$ \\
\hline Body mass index $\left(\mathrm{kg} / \mathrm{m}^{2}\right)$ & $24.2 \pm 4.6$ & $25.4 \pm 3.0$ & $25.9 \pm 4.8$ \\
\hline \multicolumn{4}{|l|}{ Rome IV diagnosis } \\
\hline $\mathrm{CIC}$ & $4(44.4)$ & $9(81.8)$ & $14(70.0)$ \\
\hline IBS-C & $5(55.6)$ & $2(18.2)$ & $6(30.0)$ \\
\hline Colonic transit time (min) & $3088 \pm 2083$ & $3524 \pm 2792$ & $3288 \pm 1838$ \\
\hline Slow-transit constipation $^{\mathrm{a}}$ & $3.0 \pm 33.3$ & $5.0 \pm 45.5$ & $7.0 \pm 35.0$ \\
\hline Stool frequency per day & $0.8 \pm 0.4$ & $0.7 \pm 0.4$ & $0.8 \pm 0.3$ \\
\hline Stool consistency per BM (BSFS 1-7) & $2.6 \pm 1.1$ & $3.1 \pm 1.1$ & $3.0 \pm 1.2$ \\
\hline Ease of passage scale per BM (1-7) & $3.9 \pm 0.7$ & $3.5 \pm 0.5$ & $3.6 \pm 0.6$ \\
\hline Number of CSBMs per day & $0.2 \pm 0.3$ & $0.2 \pm 0.2$ & $0.2 \pm 0.2$ \\
\hline Daily constipation severity $(1-10)^{\mathrm{b}}$ & $4.2 \pm 2.5$ & $4.0 \pm 1.3$ & $4.1 \pm 2.1$ \\
\hline Daily abdominal discomfort severity $(1-10)^{b}$ & $3.8 \pm 2.2$ & $3.6 \pm 1.4$ & $3.9 \pm 1.8$ \\
\hline Daily bloating severity $(1-10)^{\mathrm{b}}$ & $4.7 \pm 2.5$ & $4.4 \pm 1.4$ & $4.4 \pm 2.2$ \\
\hline
\end{tabular}

${ }^{a}$ Slow-transit constipation defined as colonic transit time $\geq 59$ hours at baseline.

${ }^{b}$ Higher values indicate increased severity of symptoms.

CIC, chronic idiopathic constipation; IBS-C, irritable bowel syndrome with constipation; BM, bowel movement; BSFS, Bristol Stool Form Scale; CSBM, complete spontaneous bowel movement.

$P$-values were calculated with the use of analysis of variance for continuous data and chi square tests for categorical data.

Data are presented as mean $\pm \mathrm{SD}$ or $\mathrm{n}(\%)$. 


\section{Results}

\section{Participants and Study Flow}

Fifty-two patients were screened for the study, as shown in Figure 2 between March 7, 2017 and March 22, 2018. Four were ineligible because of baseline abnormal lab values (positive toxicology screen or abnormal liver function tests). Six were not randomized to treatment because of loss to follow up or subject withdrawal and a further 2 were not randomized (1 due to an inability to swallow the WMC and 1 subject not meeting Rome IV IBS-C criteria during the run-in period). Recruitment was stopped prematurely due to slower than expected enrollment. Because a simple randomization scheme was used rather than block randomization, there was an unequal allocation of patients to treatment arms by chance. Demographic and baseline data of all randomized patients are shown in Table 1. The CMC, CSP01, and placebo groups were all similar regarding baseline characteristics and CTT. Two randomized patients were not included in the primary endpoint analysis, $1 \mathrm{CSP} 01$ treated (CIC group) patient dropped out because of an $\mathrm{AE}$ and the other (placebo-treated, IBS-C group) because the WMC recorder malfunctioned-providing no data on follow-up colonic transit. This left 38 total subjects, slightly below our anticipated recruitment level but above our threshold of 30 subjects to achieve $80 \%$ power to detect an effect. All patients received all doses of study medication, except for the patient who dropped out in the CSP01 group due to an $\mathrm{AE}$, who completed 27 doses.

\section{Effect of Carboxymethylcellulose and CSP01 on Colonic Transit}

There was no significant change in mean CTT by treatment $(P=0.297)$ (Table 2). In the placebo group, CTT increased by

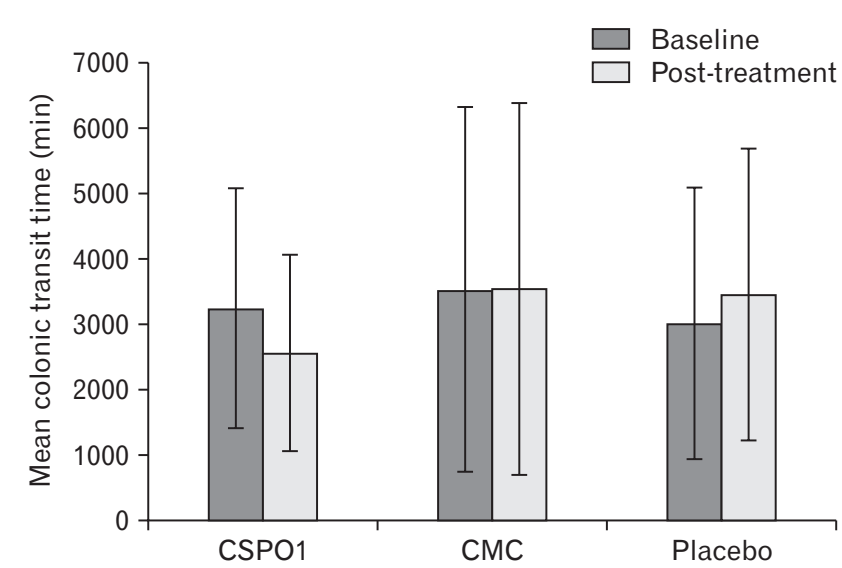

Figure 3. Baseline and post-treatment colonic transit times for all trial patients. CMC, carboxymethylcellulose.

Table 2. Change in Colonic Transit Time by Treatment Assignment and Diagnosis, Modified Intention-to-Treat Population

\begin{tabular}{|c|c|c|c|c|}
\hline \multirow{2}{*}{ Diagnosis group } & \multicolumn{3}{|c|}{ Treatment assignment } & \multirow{2}{*}{$P$-value } \\
\hline & Placebo & Carboxymethylcellulose & $\mathrm{CSP} 01$ & \\
\hline Overall (min) & $(\mathrm{n}=8)$ & $(\mathrm{n}=11)$ & $(\mathrm{n}=19)$ & \\
\hline Change in CTT & +367 & +15 & -727 & $0.297^{\mathrm{a}}$ \\
\hline Baseline CTT & $3088 \pm 2083$ & $3524 \pm 2792$ & $3288 \pm 1838$ & \\
\hline On treatment CTT & $3455 \pm 2227$ & $3539 \pm 2841$ & $2560 \pm 1505$ & \\
\hline $\mathrm{CIC}(\min )$ & $(n=4)$ & $(n=9)$ & $(\mathrm{n}=13)$ & \\
\hline Change in CTT & +1113 & -97 & -263 & 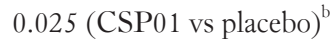 \\
\hline Baseline CTT & $3193 \pm 1728$ & $3044 \pm 2816$ & $3334 \pm 1963$ & $0.117(\mathrm{CMC} \text { vs placebo })^{\mathrm{b}}$ \\
\hline On treatment CTT & $4306 \pm 2717$ & $2611 \pm 2146$ & $2254 \pm 1235$ & \\
\hline IBS-C (min) & $(n=4)$ & $(n=2)$ & $(n=6)$ & \\
\hline Change in CTT & -92 & +2035 & +35 & $0.712(\mathrm{CSP} 01 \text { vs placebo })^{c}$ \\
\hline Baseline CTT & $2984 \pm 2665$ & $5684 \pm 1757$ & $3189 \pm 1701$ & $0.103(\mathrm{CMC} \text { vs placebo })^{c}$ \\
\hline On treatment CTT & $2604 \pm 2125$ & $7719 \pm 1085$ & $3224 \pm 1929$ & \\
\hline
\end{tabular}

${ }^{\text {a }}$-value obtained from a mixed between subject and within subject repeated measures analysis of covariance (ANCOVA) including treatment group, diagnosis (chronic idiopathic constipation [CIC] or irritable bowel syndrome with constipation [IBS-C]), gender, and age. The within subject factor crossed with each of the above was a 2-level Time factor (7 days pre-treatment or Day 15 of treatment).

${ }^{\mathrm{b}}$ Post hoc analysis using above ANCOVA model specifically among subsets of patients with CIC.

${ }^{c}$ Post hoc analysis using above ANCOVA model specifically among subsets of patients with IBS-C.

Data are presented as mean $\pm \mathrm{SD}$. 
367 minutes between baseline and end of treatment, increased by 15 minutes for CMC, and decreased by 727.4 minutes (12 hours, 7 minutes) for CSP01 (Fig. 3). A post hoc analysis demonstrated of the overall ANCOVA analysis of CTT demonstrated a complex, 3-way interaction of treatment group (placebo, CMC, or CSP01), time (before vs after treatment), and Rome diagnosis (CIC vs IBSC) at a significance level of $P=0.058$, reflecting a tendency for CTT to decline after treatment with CMC and especially CSP01, but only among patients with CIC. Additional post hoc analyses suggested that overall treatment differences (for CMC and CSP01) occurred for people with CIC, but not IBS-C $(P=0.080)$.

Among CIC patients $(n=26)$, the decline in CTT for treatment with CSP01 was significantly different from placebo $(P=0.025)$, and there was no significant difference between $\mathrm{CMC}$ and placebo (Fig. 4). Among patients with IBS-C ( $=12)$, the decline in CTT for treatment with CSP01 and CMC were not significantly different from placebo ( $P=0.712$ and $P=0.103$, respectively)(Table 2 ).

Among patients with baseline slow-transit constipation, mean CTT increased by 553.3 minutes (9 hours, 13 minutes) in the placebo group, decreased by 733.0 minutes (12 hours, 13 minutes) in
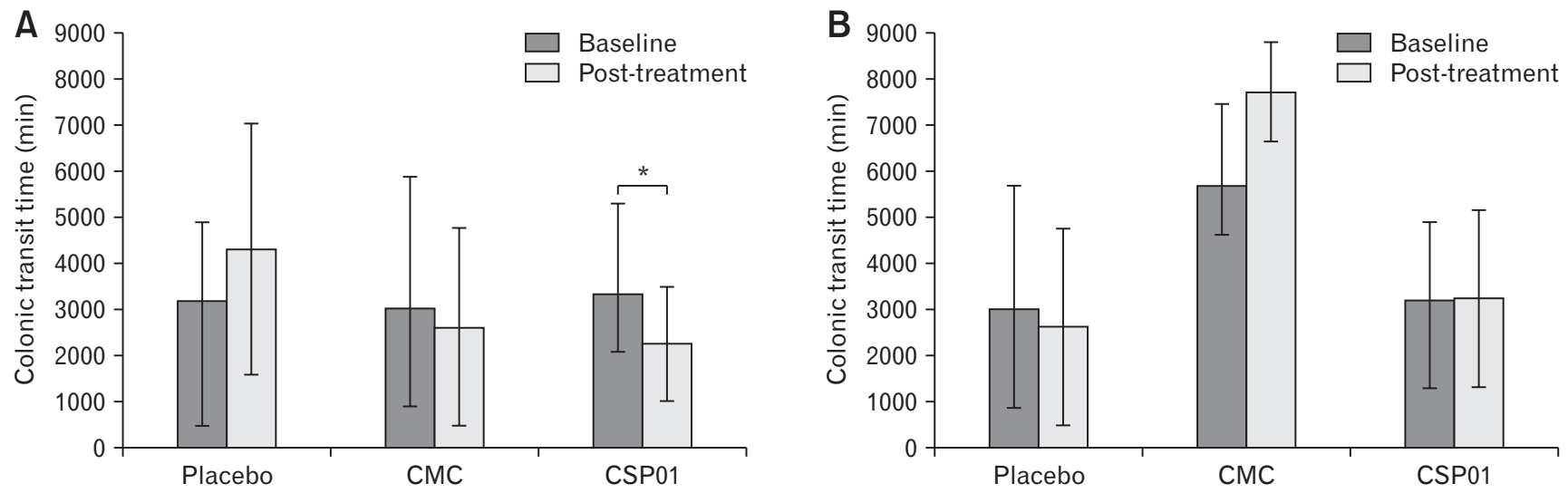

Figure 4. Baseline and post-treatment colonic transit times for patients with chronic idiopathic constipation (A) and irritable bowel syndrome with constipation (B). CMC, carboxymethylcellulose. ${ }^{*} P<0.05$.

Table 3. Effects of Carboxymethylcellulose and CSP01 on Bowel Symptoms, Patients Completing Study

\begin{tabular}{|c|c|c|c|c|c|c|c|}
\hline \multirow[t]{2}{*}{ Variables } & \multicolumn{2}{|c|}{$\begin{array}{l}\text { Placebo } \\
(\mathrm{n}=9)\end{array}$} & \multicolumn{2}{|c|}{$\begin{array}{l}\text { Carboxymethylcellulose } \\
\qquad(\mathrm{n}=11)\end{array}$} & \multicolumn{2}{|c|}{$\begin{array}{l}\text { CSP01 } \\
(n=19)\end{array}$} & \multirow[t]{2}{*}{$P$-value ${ }^{\mathrm{a}}$} \\
\hline & Baseline & Post-Tx & Baseline & Post-Tx & Baseline & Post-Tx & \\
\hline Stool frequency per day & $0.8 \pm 0.4$ & $0.9 \pm 0.6$ & $0.7 \pm 0.4$ & $0.8 \pm 0.4$ & $0.8 \pm 0.3$ & $1.0 \pm 0.4$ & 0.641 \\
\hline Stool consistency per BM (BSFS 1-7) & $2.6 \pm 1.1$ & $2.7 \pm 1.4$ & $3.1 \pm 1.1$ & $3.6 \pm 1.6$ & $3.0 \pm 1.2$ & $3.8 \pm 0.9$ & 0.509 \\
\hline Ease of passage scale per BM (1-7) & $3.9 \pm 0.7$ & $3.9 \pm 0.6$ & $3.5 \pm 0.5$ & $3.6 \pm 0.6$ & $3.6 \pm 0.6$ & $3.8 \pm 0.4$ & 0.098 \\
\hline Number of CSBMs/day & $0.2 \pm 0.3$ & $0.3 \pm 0.3$ & $0.2 \pm 0.2$ & $0.3 \pm 0.2$ & $0.2 \pm 0.2$ & $0.3 \pm 0.3$ & 0.896 \\
\hline Daily constipation severity $(1-10)^{\mathrm{b}}$ & $4.2 \pm 2.5$ & $3.9 \pm 2.5$ & $4.0 \pm 1.3$ & $3.3 \pm 1.2$ & $4.1 \pm 2.1$ & $3.5 \pm 1.8$ & 0.586 \\
\hline Daily abdominal discomfort severity $(1-10)^{\mathrm{b}}$ & $3.8 \pm 2.2$ & $3.6 \pm 2.6$ & $3.6 \pm 1.4$ & $3.1 \pm 1.4$ & $3.9 \pm 1.8$ & $3.7 \pm 1.9$ & 0.796 \\
\hline Daily bloating severity $(1-10)^{\mathrm{b}}$ & $4.7 \pm 2.5$ & $4.0 \pm 2.8$ & $4.4 \pm 1.4$ & $3.9 \pm 1.2$ & $4.4 \pm 2.2$ & $4.2 \pm 2.2$ & 0.443 \\
\hline PAC-SYM ${ }^{\mathrm{b}}$ & $15.3 \pm 7.1$ & $13.0 \pm 8.0$ & $17.5 \pm 4.6$ & $10.5 \pm 4.8$ & $18.0 \pm 8.2$ & $12.2 \pm 7.3$ & 0.422 \\
\hline PAC-QOL ${ }^{c}$ & $30.9 \pm 14.1$ & $28.6 \pm 16.1$ & $31.4 \pm 6.0$ & $21.0 \pm 4.1$ & $39.7 \pm 16.6$ & $27.7 \pm 18.4$ & 0.161 \\
\hline
\end{tabular}

${ }^{\text {a }} P$-values were calculated for effect of treatment type $\times$ time (pre-treatment vs last week of treatment) with the use of analysis of covariance with adjustment for age and sex.

${ }^{\mathrm{b}}$ Higher values indicate increased severity of symptoms.

${ }^{c}$ Higher values indicate lower quality of life.

Tx, treatment; BM, bowel movement; BSFS, Bristol Stool Form Scale; CSBMs, complete spontaneous bowel movements; PAC-SYM, Patient Assessment of Constipation-Symptoms; PAC-QOL, Patient Assessment of Constipation-Quality of Life.

One patient in the placebo-treated irritable bowel syndrome with constipation group did not have a second wireless motility capsule colonic transit time because of a technical malfunction but did complete the symptom diary and was included in this analysis. 
the CMC group, and decreased by 1425.7 minutes (23 hours, 46 minutes) in the CSP01 group. When baseline slow-transit constipation was included in the model as an additional covariate, it was not found to be significantly associated with a decrease in CTT overall $(P=0.540)$ or when IBS status was included $(P=0.120)$ across the 3 treatment groups.

\section{Effect of Carboxymethylcellulose and CSP01 on Bowel Symptoms}

There were no significant effects of treatment with either CMC or CSP01 on any bowel symptoms or disease-specific symptom severity or quality of life for the 39 patients completing the diaries and questionnaires (Table 3). Notably, 1 patient in the placebo-treated, IBS-C group did not have a second CTT because of a technical malfunction but did complete the symptom diary and was included in this analysis. Post hoc analyses for symptoms stratified by CIC (Supplementary Table 1) and IBS-C (Supplementary Table 2) demonstrated numeric improvements in bowel movement frequency, constipation symptoms, symptom severity, and quality of life for CIC. However, there was no statistically significant difference in any of the indices.

\section{Adverse Events}

There were no serious AEs, but 1 patient in the CSP01 group had to stop treatment because of an $\mathrm{AE}$, described as back pain attributed to worsening of her underlying constipation. There were no other recorded AEs.

\section{Discussion}

In this randomized, double-blind, placebo-controlled, 3-arm pilot trial of a novel superabsorbent hydrogel (CSP01) for patients with CIC and IBS-C, we found that CSP01 accelerates CTT in patients with CIC alone. Despite improvements in CTT among those with CIC, however, there were no significant improvements in stool consistency, frequency, complete spontaneous bowel movements or symptoms among patients in either group, nor any significant AEs.

Our results add additional support to the limited evidence examining the role of fiber-like compounds in the treatment of constipation. Although CSP01 has characteristics of both soluble and insoluble compounds, most traditional fibers fall predominantly into one of the two categories. Two previous trials of insoluble fiber in CIC were conflicting. One trial found increased stool frequency, softer stool consistency, and decreased difficulty in defecation among 15 patients consuming fiber-enriched rye bread, ${ }^{16}$ while the other showed no difference in straining during defecation among 9 patients treated with bran. ${ }^{17}$ In contrast, numerous trials of soluble fiber for CIC have demonstrated limited treatment benefit for constipation symptoms, but each trial suffered from significant methodological concerns. ${ }^{3}$ Importantly, none of these trials measured colonic transit as an outcome.

Our results suggest that solubility is just one aspect in the efficacy of a fiber supplement, as our active control group (CMC) demonstrated no significant reduction in CTT compared to placebo. We hypothesized 2 potential reasons why CMC failed to show benefit while CSP01 (made of 90\% CMC) treatment had a significant reduction in CTT compared to placebo. First, CMC lacks the $3 \mathrm{D}$ structure of the superabsorbent hydrogel, and therefore creates significantly lower elastic response (firmness). In an experiment simulating the in vitro gastric, small bowel, and colonic digestion of various fibers, a superabsorbent hydrogel prototype to CSP01 was found to have viscoelastic properties similar to masticated vegetables that were well in excess of those of soluble fibers such as guar gum and psyllium. ${ }^{8}$ Thus, it is possible that the inherent resistance of CSP01 to deformation applies a mechanical force to the gut wall that stimulates the mechanosensitive cells and enterochromaffin cells of the gut myenteric plexus and mucosa, respectively - initiating and maintaining the basic circuit responsible for bolus propulsion in the GI tract and improving motility. Second, because of its 3D structure, CSP01 has greater water-holding capacity than fiber products like CMC.

The improvement in CTT compared to placebo seen with CSP01 only occurred in the subset of patients with CIC and was not seen with IBS-C. Although many experts view CIC and IBS$\mathrm{C}$ as a spectrum of disease with the latter more associated with abdominal pain, the exact relationship between these 2 entities remains to be seen when using the newer Rome IV criteria utilized in the current study. Indeed, patients fulfilling the Rome IV criteria for IBS-C are thought to represent a more severe subset of Rome III IBS-C patients and therefore may suffer more visceral hypersensitivity than $\mathrm{CIC}$ patients. ${ }^{18}$ On the whole, patients with $\mathrm{CIC}$ are more likely to have delayed colonic transit than those with IBS-C, but not definitively so. ${ }^{19}$ However, there is some imaging evidence suggesting differences in response to laxatives in these 2 groups that may explain part of the differential response seen in our study. ${ }^{20}$

It should be noted that despite a significant decrease in CTT among patients with CIC treated with CSP01, there were no significant improvements noted in any specific bowel symptoms compared to placebo among those treated with CSP01. There are 
several possible reasons for the discordance between physiologic improvement and symptom-based improvements: (1) among patients with functional GI disease, the placebo effect is significantly higher than for many other GI conditions such that it can be difficult to adequately power clinical trials to detect therapeutic gain, ${ }^{21}$ and this trial was powered to detect differences in colonic transit rather than symptoms; (2) there is likely only a weak relationship between colonic transit and symptom report akin to what is seen with gastric emptying in functional dyspepsia ${ }^{22}$ and retention of radiopaque markers in constipation; ${ }^{23}$ and (3) we recruited subjects primarily from a tertiary referral population, likely selecting subjects with more severe disease and who may have been more refractory to fiber in comparison to a laxative. Nevertheless, lubiprostone, approved by the Food and Drug Administration for treatment of CIC, found an 11.7 hours reduction in CTT from baseline after 2 weeks of treatment using the same WMC methodology, consistent with the findings from the CSP01 group, and reported a significant correlation between increased bowel movement frequency and shorter CTT. ${ }^{24}$

We acknowledge several limitations. Our study was closed before randomization of 15 patients per arm as was required to attain $>90 \%$ power in our predetermined power calculations. Nevertheless, our recruited sample size of approximately 10 patients per arm achieved more than $80 \%$ power to detect a difference in colonic transit. Additionally, our positive findings occurred in a post hoc analysis of CTT according to CIC vs IBS-C, rather than an overall treatment effect for the group as a whole. Although our goal was a pure intention-to-treat analysis, we could not obtain on-treatment CTTs from 2 patients ( 1 from placebo and 1 from CSP01 group) enrolled in the study, somewhat limiting the robustness of our results. Because we did not track dietary habits of participants, we could not account for dietary fiber intake among trial participants, which could have affected our final results. Finally, we did not account for concomitant rectal evacuation disorders, which can occur in both CIC and IBS-C patients and are associated with prolonged CTTs. ${ }^{25}$

The current trial has several notable strengths. With a total of 40 subjects, this is one of the larger fiber trials in this population and one of the few trials to utilize an objective physiologic endpoint as a primary outcome. However, patients with IBS-C were underrepresented $(n=12)$. Additionally, we made use of an active control group (CMC) to give context to any positive findings seen with CSP01.

In summary, CSP01, a novel superabsorbent hydrogel, significantly decreases CTT among patients with CIC. The results of this study add a physiologic rationale to the use of superabsorbent hydrogels in patients with constipation, and CSP01 appears to be a promising agent in CIC. Further randomized trials of adequate power are warranted to determine whether CSP01 improves constipation symptoms and could become a treatment option for CIC.

\section{Supplementary Materials}

Note: To access the supplementary tables mentioned in this article, visit the online version of Journal of Neurogastroenterology and Motility at http://www.jnmjournal.org/, and at https://doi. org/10.5056/jnm20001.

Financial support: This study was funded by Gelesis Inc. Kyle Staller is supported by a career development award from the American Gastroenterological Association.

Conflicts of interest: This study was funded by Gelesis Inc. Kyle Staller has received research funding from Astra-Zeneca, Gelesis Inc, and Takeda, has served as a speaker for Shire, and has served as a consultant to Shire, Synergy, and Bayer. Braden Kuo has received research funding from AstraZeneca, Gelesis, Takeda, Alpha Waserman, Vanda, Genzyme, Covidien/Given Imaging, Vibrant Ltd, and World Care Clinical, and has served as a consultant to Actavis Pharma Inc, Biogen, Entrega, Forest Pharmaceuticals, Gelesis, Ironwood Pharmaceuticals, Takeda, Alpha Waserman, Genzyme, Shire, RTI Health Solutions, and Neurogastrx. Elaine Chiquette is an employee of Gelesis Inc. Kenneth Barshop, Abbey Bailey, and Joseph J Locascio report no disclosures.

Author contributions: Kyle Staller and Braden Kuo planned and designed the study; Abbey Bailey enrolled patients; Joseph J Locascio performed the statistical analysis; and Kyle Staller drafted the manuscript and had full access to all the data in the study and takes responsibility for the integrity of the data and accuracy of the data analysis as a guarantor of article. All authors interpreted the results and contributed to critical review of the manuscript, and approved the final version of the article, including the authorship list.

\section{References}

1. Camilleri M, Ford AC, Mawe GM, et al. Chronic constipation. Nat Rev Dis Primers 2017;3:17095.

2. Bharucha AE, Pemberton JH, Locke GR 3rd. American gastroenterological association technical review on constipation. Gastroenterology 2013;144:218-238.

3. Suares NC, Ford AC. Systematic review: the effects of fibre in the management of chronic idiopathic constipation. Aliment Pharmacol Ther 
2011;33:895-901.

4. Moayyedi P, Quigley EM, Lacy BE, et al. The effect of fiber supplementation on irritable bowel syndrome: a systematic review and metaanalysis. Am J Gastroenterol 2014;109:1367-1374.

5. Eswaran S, Muir J, Chey WD. Fiber and functional gastrointestinal disorders. Am J Gastroenterol 2013;108:718-727.

6. Timm D, Willis H, Thomas W, Sanders L, Boileau T, Slavin J. The use of a wireless motility device (SmartPill $\left.{ }^{\circledR}\right)$ for the measurement of gastrointestinal transit time after a dietary fibre intervention. Br J Nutr 2011;105:1337-1342.

7. Harris LA, Horn J, Kissous-Hunt M, Magnus L, Quigley EMM. The better understanding and recognition of the disconnects, experiences, and needs of patients with chronic idiopathic constipation (BURDENCIC) study: results of an online questionnaire. Adv Ther 2017;34:26612673.

8. Demitri C, Zohar Y, Heshmati HM, Urban LE, Aschenbach WG, Sannino A. Satiety, weight loss, and glycemic control-enhancing properties vary between functional fibers, mixed vegetables, and a novel hydrogel (Gelesis200). In European Congress on Obesity 2017, May 17-20, Porto, Portugal: P938.

9. Mearin F, Lacy BE, Chang L, et al. Bowel disorders. Gastroenterology 2016;150:1393-1407.

10. Rao SS, Kuo B, McCallum RW, et al. Investigation of colonic and whole-gut transit with wireless motility capsule and radiopaque markers in constipation. Clin Gastroenterol Hepatol 2009;7:537-544.

11. Camilleri M, Thorne NK, Ringel Y, et al. Wireless $\mathrm{pH}$-motility capsule for colonic transit: prospective comparison with radiopaque markers in chronic constipation. Neurogastroenterol Motil 2010;22:874-882, e233.

12. Heaton KW, Radvan J, Cripps H, Mountford RA, Braddon FE, Hughes AO. Defecation frequency and timing, and stool form in the general population: a prospective study. Gut 1992;33:818-824.

13. Lewis SJ, Heaton KW. Stool form scale as a useful guide to intestinal transit time. Scand J Gastroenterol 1997;32:920-924.

14. Frank L, Kleinman L, Farup C, Taylor L, Miner P Jr. Psychometric validation of a constipation symptom assessment questionnaire. Scand J Gastroenterol 1999;34:870-877.

15. Marquis P, De La Loge C, Dubois D, McDermott A, Chassany O. De- velopment and validation of the patient assessment of constipation quality of life questionnaire. Scand J Gastroenterol 2005;40:540-551.

16. Hongisto SM, Paajanen L, Saxelin M, Korpela R. A combination of fibre-rich rye bread and yoghurt containing Lactobacillus GG improves bowel function in women with self-reported constipation. Eur J Clin Nutr 2006;60:319-324.

17. Badiali D, Corazziari E, Habib FI, et al. Effect of wheat bran in treatment of chronic nonorganic constipation. A double-blind controlled trial. Dig Dis Sci 1995;40:349-356.

18. Aziz I, Törnblom H, Palsson OS, Whitehead WE, Simrén M. How the change in IBS criteria from Rome III to Rome IV impacts on clinical characteristics and key pathophysiological factors. Am J Gastroenterol 2018;113:1017-1025.

19. Siah KT, Wong RK, Whitehead WE. Chronic constipation and constipation-predominant IBS: separate and distinct disorders or a spectrum of disease? Gastroenterol Hepatol (NY) 2016;12:171-178.

20. Lam C, Chaddock G, Marciani L, et al. Colonic response to laxative ingestion as assessed by MRI differs in constipated irritable bowel syndrome compared to functional constipation. Neurogastroenterol Motil 2016;28:861-870.

21. Patel SM, Stason WB, Legedza A, et al. The placebo effect in irritable bowel syndrome trials: a meta-analysis. Neurogastroenterol Motil 2005;17:332-340.

22. Stanghellini V, Tosetti C, Paternico A, et al. Risk indicators of delayed gastric emptying of solids in patients with functional dyspepsia. Gastroenterology 1996;110:1036-1042.

23. Staller K, Barshop K, Ananthakrishnan AN, Kuo B. Number of retained radiopaque markers on a colonic transit study does not correlate with symptom severity or quality of life in chronic constipation. Neurogastroenterol Motil 2018;30:e13269.

24. Sarosiek I, Bashashati M, Alvarez A, et al. Lubiprostone accelerates intestinal transit and alleviates small intestinal bacterial overgrowth in patients with chronic constipation. Am J Med Sci 2016;352:231-238.

25. Staller K, Barshop K, Ananthakrishnan AN, Kuo B. Rectosigmoid localization of radiopaque markers does not correlate with prolonged balloon expulsion in chronic constipation: results from a multicenter cohort. Am J Gastroenterol 2015;110:1049-1055. 